ORIGINAL BREVE

\title{
Propiedades psicométricas de la escala de satisfacción con la vida en mujeres mayores activas
}

\author{
Carmen Requena Hernández ${ }^{a}$, Verónica López Fernández ${ }^{a}$, \\ René González Boto ${ }^{\mathrm{a}}$ y Tomás Ortiz Alonso \\ a Universidad de León, León, España \\ b Centro de Magnetoencefalografía Dr. Pérez Modrego, Universidad Complutense, Madrid, España
}

\section{INFORMACIÓN DEL ARTÍCULO}

\section{Historia del artículo:}

Recibido el 25 de junio de 2008

Aceptado el 9 de enero de 2009

On-line el 14 de mayo de 2009

\section{Palabras clave:}

Satisfacción con la vida

Propiedades psicométricas

Mujeres mayores

\begin{abstract}
R E S U M E N
Objetivo: El presente estudio analiza las propiedades psicométricas de la versión en castellano de la Escala de Satisfacción con la Vida.

Método: Se utilizó una muestra de 310 mujeres mayores activas, con estudios primarios y que vivesen en su domicilio habitual. La fiabilidad de la escala se examinó con el alfa de Cronbach y con un análisis factorial exploratorio.

Resultados: La fiabilidad de la escala fue de 0,75. El análisis de componentes principales mostró un único factor que explicaba el $51,32 \%$ de la variancia. El ítem 5 «Las circunstancias de mi vida son buenas» es el que explica un mayor porcentaje de variancia de los datos.

Conclusión: El análisis de fiabilidad muestra una buena consistencia interna.
\end{abstract}

(c) 2008 SEGG. Publicado por Elsevier España, S.L. Todos los derechos reservados.

\section{Psychometric properties of the satisfaction with life scale in active elderly women}

A B S T R A C T

Objective: The present study analyzes the psychometric properties of the Spanish version of the Satisfaction with Life Scale.

Methods: The sample of 310 active elderly women with primary school education and living in their own homes. The scale's reliability was examined with Cronbach's alpha and the exploratory factor analysis. Results: The reliability show a value of 0.75 . Principal components analysis showed a single factor solution accounting for $51.32 \%$ of the variance. Item 5 "The conditions of my life are excellent" explained the highest percentage of variance in the data.

Conclusión: These analyses showed acceptable internal consistency.

(c) 2008 SEGG. Published by Elsevier España, S.L. All rights reserved.

\section{Introducción}

El concepto de bienestar subjetivo incluye 2 componentes claramente diferenciados: por un lado, los juicios cognitivos sobre la satisfacción con la vida y, por otro, las evaluaciones afectivas sobre el humor y las emociones ${ }^{1}$. El presente estudio se ocupará del componente cognitivo del bienestar subjetivo.

La satisfacción con la vida se define como «un proceso de juicio mediante el cual los individuos valoran la calidad de su vida en función de sus propios criterios» ${ }^{2}$. Son los individuos mismos

\footnotetext{
* Autor para correspondencia.

Correo electrónico: tortiz@med.ucm.es (T. Ortiz Alonso).
}

quienes, al ponderar las prioridades personales de su vida, la juzgan en conjunto como más o menos satisfactoria.

El «bienestar subjetivo» (subjective well-being) ${ }^{3}$, que da nombre a este campo de investigación de la psicología, se apoya para su evaluación en la «utilidad recordada», es decir, en el grado de satisfacción global que el sujeto manifiesta respecto de un período extenso de su existencia pasada o respecto de toda ella. Se diseñó una escala multiítem ${ }^{4}$ con la idea de que se puede preguntar a las personas sobre el juicio de su vida, con el propósito de medir el concepto de satisfacción con la vida. Desde entonces, son numerosos los estudios que han analizado las propiedades psicométricas de esta escala. De éstos, algunos se han realizado fuera de España con personas mayores que viven de forma independiente $^{4-6}$ y otros, con población mayor española 
institucionalizada ${ }^{7}$. Los resultados de estas investigaciones coinciden en señalar que el ítem 5 de la escala, que hace referencia a las circunstancias que rodean la vida de una persona, es el indicador que explica un mayor porcentaje de variancia de la Escala de Satisfacción con la Vida (ESV).

En este estudio se analizan las propiedades psicométricas de la ESV con mujeres mayores activas que viven en su domicilio habitual.

\section{Método}

\section{Participantes}

La muestra estuvo compuesta por 310 mujeres con estudios primarios y un estilo de vida activo ${ }^{8}$. La captación se hizo en los centros municipales de mayores de la ciudad de León. Los criterios de inclusión fueron los siguientes: mujeres mayores de 60 años, con estudios primarios, que viviesen en su domicilio habitual solas o con cónyuge y que participasen en al menos 3 actividades de entretenimiento que se organicen en el centro municipal. Quedaron excluidas las mujeres con enfermedades psiquiátricas o neurológicas. La media de edad fue de 71,22 años (desviación estándar de 6,72 ). De las participantes, el $83 \%$ vivía solo y el resto con sus cónyuges.

\section{Instrumento}

Las participantes autocumplimentaron la versión española ${ }^{9}$ de la escala Satisfaction with Life Scale ${ }^{4}$. Es un cuestionario que mide el criterio subjetivo de satisfacción vital y que consta de 5 ítems con alternativas de respuesta múltiple que oscilan entre 5 para «muy de acuerdo» y 1 para «muy en desacuerdo». Los ítems son ítem 1 «En la mayoría de los aspectos de mi vida, es como quiero que sea»; ítem 2 «Hasta ahora he conseguido de la vida las cosas que considero importantes»; ítem 3 «Estoy satisfecho con mi vida»; ítem 4 «Si pudiera vivir mi vida otra vez, la repetiría tal y como ha sido», e ítem 5 «Las circunstancias de mi vida son buenas».

\section{Resultados}

La estructura interna del instrumento se estudió a través del análisis factorial exploratorio y se utilizó para esto el programa estadístico SPSS 15.0. Se realizó un análisis de los componentes principales (tabla 1 ).

La medida de adecuación muestral de Kaiser-Meyer-Olkin fue de 0,73 , por lo que las correlaciones entre parejas de ítems seleccionados pudieron explicarse a través del resto. La prueba de

Tabla 1

Pesos factoriales de los ítems de la Escala de Satisfacción con la Vida

\begin{tabular}{|c|c|}
\hline & $\begin{array}{l}\text { Componente } \\
\text { I }\end{array}$ \\
\hline $\begin{array}{l}\text { 1. En la mayoría de los aspectos de mi vida, es como quiero que } \\
\text { sea }\end{array}$ & 0,667 \\
\hline $\begin{array}{l}\text { 2. Hasta ahora he conseguido de la vida las cosas que considero } \\
\text { importantes }\end{array}$ & 0,631 \\
\hline 3. Estoy satisfecho con mi vida & 0,770 \\
\hline 4. Si pudiera vivir mi vida otra vez, la repetiría tal y como ha sido & 0,688 \\
\hline $\begin{array}{l}\text { 5. Las circunstancias de mi vida son buenas } \\
\mathrm{n}=310\end{array}$ & 0,811 \\
\hline Variancia (\%) & 51,32 \\
\hline Autovalor & 2,57 \\
\hline
\end{tabular}

Método de extracción: Análisis de componentes principales. Componentes extraídos: 1. esfericidad de Bartlett (test de $\chi^{2}=439,67 ; \mathrm{p}<0,001$ ) mostró la existencia de una alta dependencia entre los 5 ítems. El análisis de componentes principales extrajo un único factor, que explicaba el $51,32 \%$ de la variancia y cuyo autovalor fue 2,57 . Todos los ítems de la escala presentaron elevados pesos factoriales que oscilaron entre 0,81 para el ítem 5 y 0,63 para el ítem 2 .

Se realizó un análisis factorial confirmatorio, se utilizó el modelo estructural con el programa Amos 7.0 y se siguió el método de máxima verosimilitud para estimar la relación entre las variables. Para estimar la adecuación del modelo a los datos se emplearon los siguientes índices de ajuste: test de CMI (chi square 'ji-cuadrado'), que representa la medida fundamental del ajuste global del modelo; GFI (goodness of fit index 'índice de bondad de ajuste'); índice de Tucker-Lewis; CFI (comparative fit index 'índice de ajuste comparado'); NFI (normed fixed index 'índice de ajuste normado'); índice de la RMR (root mean residual 'raíz del residuo estandarizado cuadrático medio'), e índice del RMSEA (root mean square error of approximation 'error cuadrático medio de aproximación').

En consideración al cociente test de CMIN/DF (quotient chi square/degrees of freedom 'cociente ji-cuadrado/grados de libertad'), se observó que el valor fue de 1,15 (inferior a 2), lo que indica que el modelo presenta un buen ajuste a los datos. Además, el valor de p no fue significativo. Todo esto se corroboró con los otros índices: el GFI mostró también un buen ajuste por su cercanía al valor uno, al igual que los GFI no normativos ( $\rho$ de Tucker-Lewis), NFI (delta) y CFI. El valor obtenido en el índice de la RMR y en el índice del RMSEA fue próximo a 0 (tabla 2).

El índice de fiabilidad calculado para la escala alpha de Cronbach indica que la escala tuvo buena consistencia interna $(\alpha=0,75)$.

\section{Discusión}

Los resultados del análisis psicométrico de la versión española de la ESV presentaron buenas propiedades psicométricas en mujeres mayores activas que viven en sus casas.

El análisis factorial exploratorio mostró que la estructura unifactorial explica un porcentaje de variancia adecuado, algo superior al obtenido con individuos mayores españoles institucionalizados $^{7}$ y menor que el obtenido con individuos mayores independientes, estadounidenses ${ }^{6}$ y canadienses ${ }^{5}$.

El ítem 5 «Las circunstancias de mi vida son buenas» es el que explica el mayor porcentaje de variancia, lo que coincide con el

\section{Tabla 2}

Análisis factorial confirmatorio

\begin{tabular}{ll}
\hline Índices & \\
\hline CMI & 5,74 \\
DF & 5 \\
P & 0,33 \\
CMIN/DF & 1,15 \\
RMR & 0,052 \\
GFI & 0,98 \\
NFI (delta) & 0,97 \\
TLI (rho) & 0,99 \\
CFI & 0,99 \\
RMSEA & 0,031 \\
\hline
\end{tabular}

CFI: comparative fit index 'índice de ajuste comparado'; CMI: Chi square 'jicuadrado'; CMIN/DF: quotient chi square/degrees of freedom 'cociente jicuadrado/grados de libertad'; DF: degrees of freedom 'grados de libertad' GFI: goodness of fit index 'índice de bondad de ajuste'; NFI: normed fixed index 'índice de ajuste normado'; RMR: root mean residual 'raíz del residuo estandarizado cuadrático medio';RMSEA: root mean square error of approximation 'error cuadrático medio de aproximación'; TLI: Tucker-Lewis index 'índice de TuckerLewis'. 
estudio llevado a cabo con población mayor institucionalizada ${ }^{6}$. El hecho de que en ambos estudios los sujetos se caracterizaron por tener autonomía para la realización de las tareas en la vida diaria lleva a la reflexión de que es esta variable, y no la peculiaridad de donde viven, la que determina la satisfacción con la vida de estos sujetos. El ítem 1 «En la mayoría de los aspectos de mi vida, es como quiero que sea» también hace referencia a las circunstancias que rodean a las personas, aunque menos relevante que en población mayor institucionalizada, este ítem también tiene peso en la valoración de la satisfacción con la vida. De hecho, la consistencia de la escala no aumenta con la eliminación de ninguno de los ítems. Estos resultados podrían explicarse porque la mayoría de las mujeres de la muestra viven solas, en la etapa de la vida en la que más se valoran los vínculos socioafectivos ${ }^{10}$, frente a los usuarios de las residencias, que viven en comunidad. El ítem 3 «Estoy satisfecho con mi vida» es el segundo más válido, lo que coincide nuevamente con la muestra de individuos mayores institucionalizados y difiere con muestras de población de adolescentes ${ }^{9}$.

En cuanto al ítem 2 «Hasta ahora he conseguido de la vida las cosas que considero importantes», es el que menor porcentaje de variancia explica. Este ítem, junto con el 4 «Si pudiera vivir mi vida otra vez, la repetiría tal y como ha sido», tiene una orientación temporal hacia el pasado y explica la satisfacción con la vida por los logros obtenidos, que parecen ser mayores en las personas de más edad ${ }^{11}$.

El hecho de que los ítems que hacen referencia al presente expliquen mayor proporción de variancia que los ítems que hacen referencia al pasado podría relacionarse con el estilo de vida activo que caracteriza a los sujetos de la muestra, situación que les permite participar en la sociedad de acuerdo con sus necesidades, deseos y capacidades a lo largo de todo su ciclo vital, lo que mejora la satisfacción con la vida de las personas que envejecen ${ }^{8}$.
En cuanto a los resultados obtenidos a través del análisis factorial confirmatorio, están en consonancia con los obtenidos en los trabajos existentes con personas mayores ${ }^{5,7}$, y confirman la unidimensionalidad de la escala y que el modelo presenta un buen ajuste a los datos.

En futuras investigaciones sería interesante seguir clarificando el peso que tienen otros criterios, como el afectivo o el físico, en el indicador global de la satisfacción con la vida.

\section{Bibliografía}

1. Diener E, Lucas RE. Personality and subjective well-being. En: Kahneman D, Diener E, Schwarz N, editores. Well-being: The foundations of hedonic psychology. New York: Russell Sage Fundation; 1999. p. 213-27.

2. Pavot W, Diener E. Review of the Satisfaction with Life scale. Psychological Assessment. 1993;5:164-72.

3. Diener E. Subjective well-being. Psychollogical Bulletin. 1980;95:542-75.

4. Diener ED, Emmons RA, Larsen RJ, Griffin S. The Satisfaction with Life scale. Journal of Personality Assessment. 1985;49:71-5.

5. Blais MR, Vallerand RJ, Pelletier LG, Briere NM. L'Echelle de satisfaction de vie: Validation Canadienne-Francaise du "Satisfaction With Life Scale" (FrenchCanadian Validation of the Satisfaction With Life Scale). Canadian Journal of Behavioral Science. 1989;21:210-23.

6. Pavot W, Diener ED, Colvin CR, Sándwich E. Further validation of the Satisfaction with Life scale: Evidence for the cross-method convergence of well-being. Journal of Personality Assessment. 1991;57:149-61.

7. Pons D, Atienza FL, Balaguer I, García-Merita M. Propiedades psicométricas de la escala de satisfacción con la vida en personas de tercera edad. RIDEP. 2002;13:71-82

8. Organización Mundial de la Salud. Envejecimiento activo: un marco político. Rev Esp Geriatr Gerontol. 2002;37:74-105

9. Atienza FL, Pons D, Balaguer I, García-Merita M. Propiedades psicométricas de la escala de Satisfacción con la Vida en adolescentes. Psicothema. 2000;12: 314-9.

10. Carstensen LL, Mikels JA. At the intersection of emotion and cognition: Aging and the positivity effect. Current Directions in Psychological Science. 2005; 14:117-21.

11. Clemente A, Molero R, González F. Estudio de la satisfacción personal según la edad de las personas. Anales de psicología. 2000;16:189-98. 